\title{
Testfall für Integration
}

\section{Jugendliche mit Migrationshintergrund in der Jugendsozialarbeit}

\author{
Katharina Fournier
}

Katharina Fournier ist Referentin für Migration und Integration bei der Bundesarbeitsgemeinschaft Evangelische Jugendsozialarbeit e. V. mit Sitz in Berlin.

E-Mail fournier@bagejsa.de
Die Politik muss die Weichen neu stellen, um den Integrationserfolg der heranwachsenden Generation von Kindern und Jugendlichen mit Migrationshintergrund zu meistern.

Junge Migrantinnen und Migranten gehören zu einer wichtigen Zielgruppe der neuen Integrationspolitik des Bundes - sie sind ein Testfall für das »Integrationsland « Deutschland.

Eine erfolgversprechende Integrationspolitik für Jugendliche umfasst aus Sicht der Jugendsozialarbeit folgende Aspekte: Erstens sind sozial- und herkunftsbedingte Benachteiligungen in der schulischen und beruflichen Ausbildung von Kindern und Jugendlichen abgebaut, zweitens bestehen zielgruppenspezifische Integrationsförderangebote für Kinder, Jugendliche und ihrer Familie und drittens ist Integrationspolitik als Querschnittsaufgabe in Jugend-, Bildungs-, Arbeitsmarkt-, Sozial- und Infrastrukturpolitiken verankert. Im Folgenden wird überprüft, welchen integrationspolitischen Beitrag die Jugendsozialarbeit in diesen drei Teilaspekten heute und in Zukunft leisten kann.

\section{Angekommen in der Mitte der Gesellschaft}

Kinder und Jugendlichen mit Migrationshintergrund sind in der Mitte der Gesellschaft und somit in den gesellschaftlichen und politischen Diskursen angekommen - mit allen für sie daraus erwachsende Vor- und Nachteile: Vorteilhaft für sie wirkt sich die längst überfällige politische Aufmerksamkeit aus, indem ihre Belange zukünftig stärker berücksichtigt werden. Immerhin machen junge Migrantinnen und Migranten mit 27,8 Prozent schon über ein Viertel der bildungsrelevanten Bevölkerung der unter 25-Jährigen aus. Bei den unter Sechsjährigen hat laut Mikrozensus jedes drit- te Kind in Deutschland einen Migrationshintergrund $(33 \%)$ - eine Tendenz, die für das ganze Bundesgebiet zukunftsweisend ist. Die ostdeutschen Bundesländer, die heute einen vergleichsweise geringen Anteil von Jugendlichen mit Migrationshintergrund aufweisen (unter 10\%), werden von den Erfahrungen in Hamburg, Bremen (über 40\%), Nordrhein-Westfalen, Hessen und Berlin (durchschnittlich $30 \%$ ) profitieren können (vgl. Statistisches Bundesamt 2006).

Nachteilig wirkt sich die gesteigerte öffentliche Aufmerksamkeit für den einzelnen Jugendlichen aus, wenn der »Migrationshintergrund « als Ursache von Gewaltbereitschaft, Schulversagen und Parallelgesellschaft herangezogen wird. Die politische Aufmerksamkeit geht mit einem notwendigen, aber oftmals defizitorientierten öffentlichen Diskurs einher, der die Sichtweise auf die einzelne junge Migrantin und den einzelnen jungen Migranten verzerren kann. Um herkunftsbezogene Stigmatisierungen zu vermeiden, ist eine differenzierte und wissenschaftlich fundierte Diskussion umso wichtiger, die auch die Ressourcen der Jugendlichen aufgreift.

\section{Integration durch Jugend- sozialarbeit: Bildungschancen verwirklichen}

In Einrichtungen der Jugendsozialarbeit gehören junge Migrantinnen und Migranten schon seit vielen Jahren zu einer der wichtigsten Zielgruppen. Ob in Angeboten der mobilen Jugendarbeit, der Jugendberufshilfe oder der schulbezogenen Jugendsozialarbeit: Kinder und Jugendliche mit Migrationshintergrund nehmen die Angebote außerhalb der Schule und der betrieblichen Ausbildung oft überdurchschnittlich häufig wahr. Im Ausbildungsjahr 2004/2005 hatten 18 Prozent der Jugendlichen, die über Maßnahmen der Jugendsozialarbeit erreicht 
wurden, einen Migrationshintergrund (BAG EJSA 2006: 5). Ein vergleichsweise hoher Prozentsatz, den viele andere Einrichtungen der Kinder- und Jugendhilfe nicht erreichen. Dass die Jugendsozialarbeit über diesen vermeintlich privilegierten Zugang verfügt, ist auf ihren ganzheitlichen Bildungs- und Förderungsauftrag zurückzuführen:

Die Jugendsozialarbeit hat den Auftrag jeden jungen Menschen in seiner schulischen, beruflichen und gesellschaftlichen Integration zu unterstützen, um soziale Benachteiligungen und individuelle Beeinträchtigungen auszugleichen. Bei Kin-
Für die schulische Integration verfügen die schulbezogene Jugendsozialarbeit und die Schulsozialarbeit über bewährte Konzepte. Hierzu gehören beispielsweise Schulsozialarbeiterinnen und Schulsozialarbeiter als Vermittler zwischen Schulen und Elternhaus oder Angebote zum Nachholen des Schulabschlusses.

Neben dem Schulabschluss stellt der erfolgreiche Übergang in die Ausbildung die zweite Schwelle dar: Trotz vergleichbarer Noten sind jungen Migrantinnen und Migranten häufig durch diskriminierende Auswahlpraktiken von Betrieben benachteiligt, was sich in der stetig sin-

\section{"Junge Migrantinnen und Migranten stellen bereits über ein Viertel der bildungsrelevanten Bevölkerung der unter 25-Jährigen"}

dern und Jugendlichen aus Migrationsfamilien »kumulieren sich Benachteiligungen aus sozial niedrigem Status, ethnischer Zugehörigkeit und dem sozialen Umfeld « (Boos-Nünning 2005: 177). Junge Migrantinnen und Migranten verfügen im Vergleich zu Gleichaltrigen ohne Migrationshintergrund im Durchschnitt nicht über die gleiche Ausstattung an »Kapital « in Form von Geld, Bildungszugängen, Information und Kommunikation (vgl. DJI 2006: 11). Der klassische Effekt sozialer Ungleichheit wirkt bei ihnen stärker und dementsprechend sind sie bei den niedrigen Schulabschlüssen häufiger vertreten und öfter von $\mathrm{Ar}$ beitslosigkeit betroffen.

Die starke Abhängigkeit des Bildungserfolgs von der sozialen Herkunft ist in allen einschlägigen wissenschaftlichen Untersuchungen der vergangenen Jahre (vgl. Konsortium Bildungsberichterstattung 2006) umfassend dokumentiert und kritisiert worden. Dieser Umstand erklärt aber auch, warum sie häufiger in Angeboten der Jugendsozialarbeit vertreten sind: Die Jugendsozialarbeit eröffnet ihnen im Bedarfsfall alternative Bildungsformen. Diese sind bei jungen Migrantinnen und Migranten aufgrund der strukturellen Benachteiligung besonders im Bereich schulischer und beruflicher Integration nachgefragt. kenden Ausbildungsquote junger Migrantinnen und Migranten widerspiegelt. Hier bieten die außerbetrieblichen Ausbildungsgänge der Jugendberufshilfe zumindest übergangsweise eine alternative Ausbildungsperspektive und ermöglichen somit im besten Fall anschließend den Übergang in die Arbeitswelt. In jedem Fall sind sie eine weitere Qualifizierung und bieten durch ihre pädagogische Begleitung eine individuelle Förderung der Jugendlichen.

Die schulbezogene Jugendsozialarbeit und die Jugendberufshilfe sind die beiden Arbeitsfelder der allgemeinen Jugendsozialarbeit, in denen junge Migrantinnen und Migranten überdurchschnittlich stark vertreten sind. Dabei kann die Jugendsozialarbeit weder die versäumten Schulabschlüsse noch die fehlenden Ausbildungsplätze ersetzen. Sie ermöglicht aber einen gleichberechtigten Zugang zu Bildung, Ausbildung und gesellschaftlicher Mitwirkung durch außerschulische Bildungsangebote.

\section{Jugendmigrationsdienste: ziel- gruppenspezifische Integrations- hilfen der Jugendsozialarbeit}

Neben bildungspolitischer Weichenstellung sind zielgruppenspezifische Inte- grationsangebote, die eine individuelle Begleitung junger Migrantinnen und Migranten über einen längeren Zeitraum ermöglichen, ein weiterer Aspekt einer jugendgerechten Integrationspolitik.

Mit den Jugendmigrationsdiensten haben sich spezialisierte Fachdienste der Jugendsozialarbeit etabliert, die sich mit ihren Angeboten explizit an jungen Menschen mit Migrationshintergrund im Alter zwischen 12 und 27 Jahren richten. Die Jugendmigrationsdienste wenden sich sowohl an Jugendliche, die gerade erst nach Deutschland gekommen sind, als auch an junge Migrantinnen und Migranten in konkreten Krisensituationen, die schon länger in Deutschland leben. Für erwachsene Migrantinnen und Migranten sowie Familien gibt es das ebenfalls vom Bund geförderte Beratungsangebot der Migrationserstberatung.

Die Jugendmigrationsdienste werden im Auftrag des Bundesjugendministeriums von den Trägern der Jugendsozialarbeit (Arbeiterwohlfahrt, Bundesarbeitsgemeinschaft Katholische Jugendsozialarbeit, Bundesarbeitsgemeinschaft Evangelische Jugendsozialarbeit, Internationaler Bund, Deutscher Paritätischer Wohlfahrtsverband, Deutsches Rotes Kreuz) durchgeführt. Bundesweit gibt es zurzeit 369 Jugendmigrationsdienste. Auf der gemeinsamen Internetseite www.jugendmigrationsdienste.de können die Jugendlichen schnell und unkompliziert den nächsten Jugendmigrationsdienst in ihrer Nähe finden.

Aufgabe des Jugendmigrationsdienstes ist es, junge Migrantinnen und Migranten in ihrem Integrationsprozess zu unterstützen. Dabei treffen sie auf professionelle pädagogische Fachkräfte, die sich auf die sprachlichen, beruflichen und sozialen Integrationsangebote vor Ort und die Arbeit mit Jugendlichen spezialisiert haben. Viele der Fachkräfte in Jugendmigrationsdiensten haben selbst einen Migrationshintergrund und sprechen die Sprachen der Herkunftsländer, aus denen die meisten der Jugendlichen kommen.

Im Mittelpunkt der Arbeit der Jugendmigrationsdienste steht die individuelle Begleitung der Jugendlichen mittels Case Management. Dabei erarbeiten die Jugendlichen gemeinsam mit der Fachkraft einen individuellen Integrationsplan, in dem ausgehend von den Kompetenzen der Jugendlichen die schulischen, beruflichen und persönlichen Integrations- 
schritte vereinbart werden. Neben der individuellen Begleitung sind die Jugendmigrationsdienste für die sozialpädagogische Begleitung der Integrationskurse zuständig und bieten offene Gruppenangebote, wie Internetcafés oder Sportgruppen an. Durch die Gruppenangebote können die Jugendlichen Gleichaltrige treffen, die in einer ähnlichen Situation sind, ihre Bildungschancen verbessern, ihre Einstellungen diskutieren und sich stabilisieren.

Als Fachdienst für junge Migrantinnen und Migranten vermittelt der Jugendmigrationsdienst an der Schnittstelle zwischen Schule, Elternhaus, Ausbildung und Arbeitsamt, Jugendamt und Sportverein und arbeitet in einem Netzwerk mit den wichtigsten Einrichtungen im Gemeinwesen zusammen. Seine Mittlerfunktion und sein Fachwissen sind gefragt, wenn es darum geht, die interkulturelle Öffnung voranzutreiben und die Interessen junger Migrantinnen und Migranten bei der Planung von Vorhaben angemessen zu berücksichtigen.

Der Jugendmigrationsdienst ist im Bereich der Jugendmigrationsarbeit das wichtigste Integrationsangebot für Ju-

\section{Literatur}

Boos-Nünning, Ursula (2005): Kinder und Jugendliche mit Migrationshintergrund: Armut und soziale Deprivation, in: Zander, Martina (Hg.): Kinderarmut. Einführendes Handbuch für Forschung und soziale Praxis, Wiesbaden 2005, S. 161-180.

Bundesministerium für Familie, Senioren, Frauen und Jugend (BMFSFJ, 2006): Zwölfter Kinder- und Jugendbericht. Bericht über die Lebenssituation junger Menschen und die Leistungen der Kinder- und Jugendhilfe in Deutschland, Berlin.

Deutsches Jugendinstitut (DJI 2006): DJI Bulletin 76: Jugend und Migration, 3/2006, Deutsches Jugendinstitut e.V., München.

Konsortium Bildungsberichterstattung (2006): Bildung in Deutschland. Ein indikatorengestützter Bericht mit einer Analyse zu Bildung und Migration, Bielefeld.

Statistisches Bundesamt (2006): Leben in Deutschland. Haushalte, Familien und Gesundheit. Ergebnisse des Mikrozensus 2005, Wiesbaden. gendliche mit Migrationshintergrund in Deutschland. Mit ihrem zielgruppenspezifischen Angebot erreichen sie die Jugendlichen frühzeitig und unterstützen mit ihrer individuellen Begleitung und Vermittlungsleistung den erfolgreichen Integrationsprozess. Der niedrigschwellige Zugang über das jugendspezifische Profil und die Freiwilligkeit des Angebots sind dabei zwei einfache, aber wesentliche Erfolgsfaktoren, die auch für andere Einrichtungen beispielhaft sein könnten.

\section{Querdenken: Integration als Querschnittsaufgabe}

Der Erfolg der Jugendsozialarbeit bei sozialisations- und bildungsbegleitenden Integrationshilfen wird nicht zuletzt davon abhängen inwieweit es gelingt, Integrationspolitik als Querschnittsaufgabe zu verankern. Voraussetzung hierfür sind Integrationskonzepte, die an den Spitzen von Politik, Verwaltung und von Trägern der Jugendsozialarbeit ansetzen. Gefragt sind Konzepte, die sich sozialräumlich orientieren und sowohl vorhandene als auch neue Einrichtungen und deren Erfahrungen einbeziehen. Eine entsprechende strategische Steuerung auf politischer Ebene, wie sie beispielsweise in Stuttgart durch die Stabsstelle für Integrationspolitik geschaffen wurde und die Ausstattung mit zusätzlichen Ressourcen sind wichtige strukturelle Erfolgfaktoren. Zur Stärkung der Verbindlichkeit sollten gemeinsame Ziele und eine regelmäßige Integrationsberichterstattung als Mindeststandards zur Evaluierung des Integrationsstands vereinbart werden.

Das skizzierte Interesse für die integrationspolitischen Bedarfe junger Menschen spiegelt sich nicht zuletzt darin wieder, dass neben dem Bund mit dem Nationalen Integrationsplan auch alle Bundesländer ein mehr oder weniger differenziertes Integrationskonzept vorgelegt haben. Der nächste Schritt ist nun, die Umsetzung von Integrationskonzepten auf kommunaler und regionaler Ebene zu fördern.

Im Hinblick auf die Bildungspolitik wird ausschlaggebend sein, inwiefern es auf kommunaler Ebene gelingt, Brücken $\mathrm{zu}$ bauen zwischen den verschiedenen Angeboten und Einrichtungen. Denn der Abbau von herkunfts- und sozialbedingten Bildungsbenachteiligungen kann nicht allein durch die interkulturelle Qualifizierung der Lehrkräfte erreicht werden. Vielmehr sollten kommunale Bildungsstrategien verfolgt werden, die formale, non-formale und informelle Lernwelten miteinander verbinden und die unterschiedlichen Kompetenzen bezüglich Bildung, Qualifizierung und Beschäftigung aufeinander abstimmen. Hierbei ist die kultursensible Gestaltung der Angebote, die Einbeziehung der Eltern und eine konsequente interkulturelle Öffnung aller beteiligten Einrichtungen Voraussetzung für den Bildungserfolg der jungen Migrantinnen und Migranten.

Aufgabe der Organisationen der Jugendsozialarbeit wird es sein, ihre Expertise einzubringen, die Interessen der Jugendlichen zu erfassen und zu vermitteln und als Multiplikator zu wirken. Durch ihre föderal organisierten Strukturen hat die Organisation im Kooperationsverbund Jugendsozialarbeit (vgl. Seite 61) die Möglichkeiten auf den unterschiedlichen Entscheidungsebenen darauf hinzuwirken, dass Integrationspolitik auf kommunaler, Landes- und Bundesebene als Querschnittsaufgabe verankert wird.

\section{Die Neuauflage!}

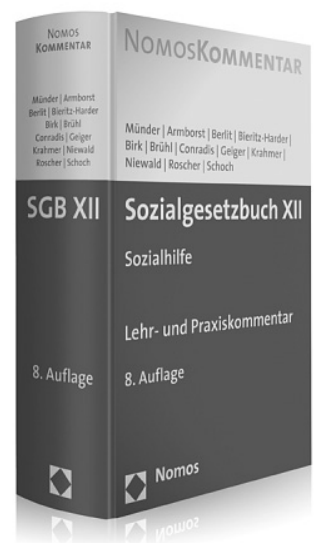

\section{Sozialgesetzbuch XII}

Sozialhilfe

Lehr- und Praxiskommentar

Von Prof. Dr. Johannes Münder u. a.

8. Auflage 2008, 1.017 S., geb.,

44,-€, ISBN 978-3-8329-2930-5

Nomos 